\title{
Simultaneous Localization and Mapping Using Rao-Blackwellized Particle Filters in Multi Robot Systems
}

\author{
Luca Carlone • Miguel Kaouk Ng • Jingjing Du • \\ Basilio Bona $\cdot$ Marina Indri
}

Received: 30 October 2009 / Accepted: 28 July 2010 / Published online: 14 August 2010

(C) The Author(s) 2010. This article is published with open access at Springerlink.com

\begin{abstract}
In this paper we investigate the problem of Simultaneous Localization and Mapping (SLAM) for a multi robot system. Relaxing some assumptions that characterize related work we propose an application of Rao-Blackwellized Particle Filters (RBPF) for the purpose of cooperatively estimating SLAM posterior. We consider a realistic setup in which the robots start from unknown initial poses (relative locations are unknown too), and travel in the environment in order to build a shared representation of the latter. The robots are required to exchange a small amount of information only when a rendezvous event occurs and to measure relative poses during the meeting. As a consequence the approach also applies when using an unreliable wireless channel or short range communication technologies (bluetooth, RFId, etc.). Moreover it allows to take into account the uncertainty in relative pose measurements. The proposed technique, which constitutes a distributed
\end{abstract}

L. Carlone $(\bowtie) \cdot$ M. Kaouk Ng

CSPP, Laboratorio di Meccatronica, Politecnico di Torino,

Corso Duca degli Abruzzi, 24, 10129, Torino, Italy

e-mail: luca.carlone@polito.it

M. Kaouk Ng

e-mail: miguel.kaoukng@polito.it

J. Du $\cdot$ B. Bona $\cdot$ M. Indri

Dipartimento di Automatica e Informatica, Politecnico di Torino,

Corso Duca degli Abruzzi, 24, 10129, Torino, Italy

J. Du

e-mail: jingjing.du@polito.it

B. Bona

e-mail: basilio.bona@polito.it

M. Indri

e-mail: marina.indri@polito.it 
solution to the multi robot SLAM problem, is further validated through simulations and experimental tests.

Keywords Mobile robots $\cdot$ Multi robot SLAM • Rao-Blackwellized particle filters

\section{Introduction}

Recent advances in mobile robotics have allowed autonomous systems to be involved in many successful applications including planetary exploration, search and rescue, surveillance, and other service scenarios [1]. For the purpose of successfully accomplishing a generic task, a main prerequisite for a mobile robot, deployed in an unknown area, is the capability of autonomously navigate, exploiting the information acquired through the joint estimation of its positions and a model of the surrounding environment. The problem of estimating both the robot pose and the environment representation is usually defined Simultaneous Localization and Mapping (SLAM), and its development and application have attracted large attention from the robotic community over the last decades. While the maturity of SLAM in single robot scenarios is recognized in many recent works [2-4], a challenging issue is to extend these approaches to multi robot scenarios in order to enhance autonomous exploration and large scale SLAM. Although improving efficiency and robustness of operation, multi robot scenarios introduce several sources of complexity requiring a bigger effort in designing probabilistic filters for the estimation of the SLAM posterior of different robots by fusing the prioceptive and the eteroceptive information acquired by each teammate. Compared to a single robot scenario, several challenges arise, including: 1) distributed posterior estimation from the available data gathered by different robots; 2) the influence of limited bandwidth and sensing range, connected to the use of unreliable wireless communication channels; 3) team coordination and need of shared world representation; 4) complexity and memory requirements in dependence of the number of robots and map size; 5) estimation in intrinsically dynamic environment.

In the following we will discuss some relevant aspects connected to the points mentioned above, introducing a simple taxonomy of the multi robot SLAM problem, consistent with the recent literature on this topic. This taxonomy will be further enriched in Section 2, in which related work on the estimation of SLAM posterior of a team of robots will be reported. A crucial role in the estimation process is played by the prior knowledge available for solving SLAM. When relative initial poses of the robots are exactly known the problem easily extends from the single robot SLAM techniques [5,6]. On the other hand in case of unknown initial correspondence of robot locations (no prior information on relative initial positions) the SLAM estimation and the information fusion is often challenging. A further distinction can be applied, classifying the approaches for solving SLAM in centralized and distributed techniques. In centralized approaches, all the information acquired by the teammates (commands and measurements) are gathered to a central node, that performs computation over the whole team posterior. This solution is often undesirable since it requires a stable communication among all the robots at each time, and this prerequisite cannot be met when using an unreliable wireless channel, 
prone to failures and quickly saturated by the large amount of information gathered to the central node. As a consequence distributed approaches are required, relaxing the strong assumption that all the team has to remain inside the communication range of the central node and improving the robustness with respect to a centralized technique in which the system cannot perform estimation in case of failures of the central node. Distributed approaches allow the robots to build their own world representation using only local information and the data exchanged with the teammates in the communication range. Although the computation remains local, the outcome of the estimation over the map model is expected to be as shared as possible, in order to enhance team coordination. For example in cooperative information gainbased exploration [7] the robots are supposed to have a shared representation of the surrounding world in order to allow coordinated actions. If each robot has its own map, the information gain of each robot cannot be easily compared with the ones of the other teammates and also the task allocation becomes challenging, since there is no common reference frame on which target assignment can be performed. Finally, the technique used to solve SLAM is required to be scalable (in terms of memory and computational complexity) and robust to dynamic environments, since the team travels in the same scenario and each robot should build a consistent map although facing the teammates that represent moving obstacles.

As witnesses of the attention paid by the robotic community to the mentioned challenges, there is a large literature in the field of multi robot SLAM (see Section 2). In such a scenario, the use of Rao-Blackwellized Particle Filters (RBPF), which are probably the most used approach to estimate metric maps in single robot scenarios, found relatively few contributions, since the high dimensionality of the state in the estimation process prevents efficient solutions unless ad-hoc techniques are applied. We here propose an extension of the grid-based SLAM to the multi robot context for the purpose of building a metric representation of the environment by means of a distributed estimation process. Relaxing the strict assumptions that characterize related work, our approach is suitable for a realistic setting in which the relative initial positions of the robots are unknown and robots can communicate only within a limited communication range. Data exchange among robots is only required when a meeting among robots occurs, whereas single robot RBPF-SLAM is performed when no communication is available. A further contribution of this work is the possibility of taking into account the uncertainty in relative measurements during rendezvous, which is shown to have a major influence on the quality of the estimation process, and cannot be neglected in most of real world applications.

The article is organized as follows. An overview of the state-of-the-art approaches to multi robot SLAM is presented in Section 2. In Section 3 our approach to RBPF-SLAM is described in deep and clarified through practical examples. Then in Section 4 we present results from simulations and real tests. Conclusions are drawn in Section 5.

\section{Related Work}

Multi robot SLAM is an active research field and many efforts were devoted to find suitable filtering techniques able to deal with a team of robots performing cooperative exploration in unknown environments. As mentioned above, the problem 
imposes harder constraints than single robot SLAM, so many authors proposed adhoc extensions of the bayesian framework in order to adapt it to distributed multi agent estimation. As for the study of single robot SLAM problem, it is possible to distinguish feature-based representations, in which the map model is expressed by means of landmarks in the environment, from metric representations, which provide a fine-grained model of the scenario. In the rest of this section we describe relevant approaches for multi robot SLAM based on both metric and landmarkbased representations.

In order to face the challenge of integrating the information collected by different robots in a consistent representation, research works proposed the use of the Extended Kalman Filter (EKF) [8], to jointly estimate robots and landmarks posterior included in an augmented state space. In [9], multi robot SLAM problem is addressed, relaxing the hypothesis of known initial correspondence. Further study on distributed estimation by means of EKF can be found in [10] and [11]. Since the EKF involves a complexity which is quadratic in the state space dimension, Thrun [12] formulated the landmark-based multi robot SLAM using the Sparse Extended Information Filter (SEIF), which constitutes an efficient solution of the SLAM problem in the information space. Taking advantages from the structure of the filter, SEIF approach can be performed in a distributed manner, overcoming the non easily decomposable structure of the EKF.

As metric maps are characterized by higher resolution and allow finer planning and exploration, many authors proposed to go over the less detailed landmarkbased representations, by exploiting the idea of sub-map approach in order to build a graph-like topological map, in which vertices represent local metric maps and edges describe relative poses of adjacent local maps. These algorithms are shown to be extendable to the multi robot SLAM $[2,13]$. Unfortunately, when the number of features in the environment increases, the computation cost becomes unsustainable. Other approaches to multi robot SLAM with metric world models are based on feature matching applied to grid maps. In a recent work [14], Carpin borrowed some concepts from image processing, applying line detection algorithms and Hough transform to the original metric map. Exploiting spectral information the author computes a set of possible transformations, i.e., rotation and translation, needed to consistently merge the maps of two robots. In [15], instead, a manifold map structure is applied to multi robot SLAM scenario, by adopting a maximumlikelihood estimation algorithm [16] for the manifold representation. Because of the centralized processing, the communication issue should be carefully considered, and many drawbacks arise reducing the potential number of teammates in the system.

The multi robot SLAM problem turns out to be even more challenging when using Rao-Blackwellized Particle Filters. In such a case each robot carries on several map hypotheses (one for each particle) and it is not straightforward to merge this large amount of data among the teammates. In [17], Howard distinguishes the case of known initial correspondences from the one in which robots are deployed without any a-priori information on their relative poses. After detailing his solution to the former case, Howard focuses the attention on the latter, proposing to solve the multi robot posterior estimation by augmenting the state space of each robot with the trajectories of the other robots in the team. When two robots meet at occasional rendezvous, each agent initializes a new set of samples, which are in charge of 
approximating the trajectory posterior of the met teammate. After the meeting each robot continues to iterate both filters, by using its own sensor measurements and the data communicated by the other robot. Once all teammates have met, each robot behaves as a central node, performing estimation of the SLAM posterior over the whole team. Although this work is strictly related to the proposed approach, some limitations reduce its effectiveness. Howard assumed for his approach a stable wireless connection that allows the robots to exchange every command and measurement from sensors among all robots at each sampling time. Moreover, a careful study of this technique reveals that the approach is substantially centralized and each robot uses a fixed particle set size to estimate over an augmented state space, although this has a large impact on the consistency of the map as we underlined in our previous work [18]. Finally the error on relative measurements is neglected and, as shown in Section 4, this approximation can lead to poor results in real scenarios. Further comparisons with the approach of [17] are reported in Section 4.2.

\section{Multi Robot RBPF-SLAM}

\subsection{Problem Statement}

We consider the case in which a team of $N$ robots, each one equipped with laser scanner, camera and odometric pose estimation, travels in an unknown indoor scenario, with the primary aim of building a consistent metric representation of the environment. The robots start from unknown initial poses (relative positions between robots are unknown too) and each agent of the formation has local knowledge of the surrounding environment (given by laser and camera). Moreover communication among agents is possible only within a maximum distance $r$, by means of an unreliable wireless channel. Without loss of generality we assume that a rendezvous event is only between two robots at a time.

Each robot is asked to obtain a metric map of the environment since the team is assumed to work in a highly symmetric scenario in which it is tricky to solve the correspondence problem of a landmark-based representation. This map should be as shared as possible among the teammates in order to enhance team coordination and allow active rendezvous and loop closing procedures.

\subsection{Notation}

In this subsection we introduce the basic notation used in the paper. We assume that each robot of the team is denoted with a unique identification number, so we will call robot $i$ the $i$-th robot of the formation. The pose of the robot $i$ at time $t$, expressed in the reference frame $\mathcal{R}_{i 0}$ is $p_{i, t}^{i 0} \doteq\left[\begin{array}{lll}x_{i, t}^{i 0} & y_{i, t}^{i 0} & \theta_{i, t}^{i 0}\end{array}\right]^{\top}$, where $x_{i, t}^{i 0}$ and $y_{i, t}^{i 0}$ describe robot position and $\theta_{i, t}^{i 0}$ represents its orientation. We further define the homogeneous coordinates of the robot, obtained from the pose $p_{i, t}^{i 0}$ by augmenting the vector with

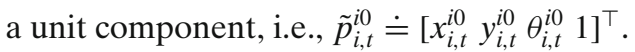

Let $u_{i, t}^{i 0}$ be the column vector containing the odometric estimate of the robot pose $p_{i, t}^{i 0}$, sampled at the $t$-th discrete time. The odometric trajectory up to time $t$ can be 
described by the following matrix containing subsequent odometric poses, assumed by the robot, ordered by columns:

$$
u_{i, 1: t}^{i 0} \doteq\left[\begin{array}{llll}
u_{i, 1}^{i 0} & u_{i, 2}^{i 0} & \cdots & u_{i, t}^{i 0}
\end{array}\right]=\left[\begin{array}{cccc}
x_{i, 1}^{i 0} & x_{i, 2}^{i 0} & \cdots & x_{i, t}^{i 0} \\
y_{i, 1}^{i 0} & y_{i, 2}^{i 0} & \cdots & y_{i, t}^{i 0} \\
\theta_{i, 1}^{i 0} & \theta_{i, 2}^{i 0} & \cdots & \theta_{i, t}^{i 0}
\end{array}\right] .
$$

When sampling the odometric pose, robot $i$ also acquires measurements from the laser scanner. The latter provides a distance measurement for $\ell$ discrete angles within laser field of view. In the example of Fig. 1 the laser returns distance information for angles in the interval $\left[\theta_{i, t}^{i 0}-\pi / 2, \theta_{i, t}^{i 0}+\pi / 2\right]$ with resolution of five degrees. We can summarize all the measurements acquired before time $t$ in the following matrix:

$$
z_{i, 1: t} \doteq\left[\begin{array}{llll}
z_{i, 1} & z_{i, 2} & \cdots & z_{i, t}
\end{array}\right] \in \mathbb{R}^{\ell \times t},
$$

in which the $t$-th column contains the distance measurements of the scan acquired at time $t$.

It is worth noticing that the distance data are expressed in polar coordinates relative to the robot reference frame, whereas they can be reported to a global reference frame introducing the information on the pose at the corresponding time steps. The information acquired by each robot can be packed in a unique variable containing prioceptive and eteroceptive data, that will be used for SLAM posterior estimation:

$$
d_{i, 1: t}^{i 0} \doteq\left[\begin{array}{c}
u_{i, 1: t}^{i 0} \\
z_{i, 1: t}
\end{array}\right]=\left[\begin{array}{llll}
u_{i, 1}^{i 0} & u_{i, 2}^{i 0} & \cdots & u_{i, t}^{i 0} \\
z_{i, 1} & z_{i, 2} & \cdots & z_{i, t}
\end{array}\right] .
$$

Finally we denote a meeting with the time stamps of the robots involved, respectively $t_{j, k}^{i}$ and $t_{i, k}^{j}$ (as they can be different for lack of synchronization), where the superscript indicates the robot from which the time stamp is sampled, the first subscript corresponds to the $i d$ of the met teammate and the last subscript indicates that the time is referred to the $k$-th rendezvous event. For sake of simplicity

Fig. 1 Range measurements from laser scanner at time $t$. The length of each radius is an entry of the vector $z_{i, t}$

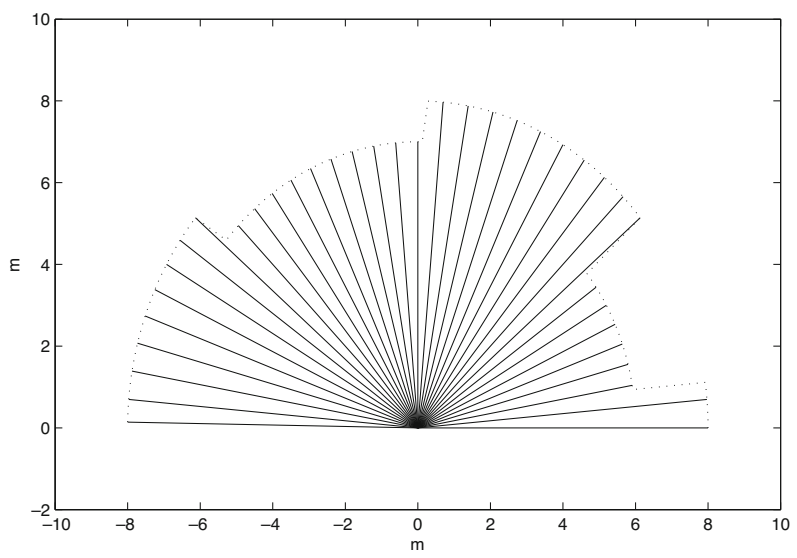


subscripts are omitted when they can be easily inferred from the context. When it is not specified, vectors are intended to be column vectors.

\subsection{Approach Overview}

The approach we propose is an efficient extension of RBPF single robot SLAM. Before and after each rendezvous the robots of the team perform their estimation using RBPF-SLAM, following the path drawn by [19] and [20]. When a rendezvous occurs, a simple procedure allows to fuse the information in an effective and distributed fashion. This multi robot approach can be summarized in three phases:

- Data exchange: the two robots, namely $i$ and $j$, exchange the data acquired since the last meeting (or since the beginning, if it is the first meeting between the two robots) to the rendezvous instant; in order to minimize the data to be exchanged, robot $i$ communicates only the odometric data and the corresponding laser scanner measurements, i.e., $d_{i, t_{j, k-1}^{i}: t_{j, k}^{i}}^{i}$, whereas $j$ communicates $d_{j, t_{i, k-1}^{j}: t_{i, k}^{j}}^{j 0}$;

- Reference frame transformation: from the information received by the teammate, and using relative pose measurements, each robot suitably roto-translates the data received in its own reference frame;

- Estimation on virtual data: once the data are roto-translated, they are used to estimate SLAM posterior as they were due to laser and odometric measurements acquired by the robot itself. RBPF estimate the posterior from received data, using suitable process models with the corresponding uncertainties.

Finally, after the filtering of received data is complete, the particles within the filters restart from their poses before the meeting, and continue the estimation process, according to grid-based RBPF-SLAM.

The approach is detailed in the following subsections, describing each phase in chronological order.

\subsection{Team Setup and Single Robot RBPF-SLAM}

The robots start from unknown initial poses and they begin to acquire information from the surrounding environment and from the prioceptive sensors. Robot $i$ fixes the reference frame in its own initial pose, namely $\mathcal{R}_{i 0}$. Each agent can start at a generic instant of time and no synchronization among the teammates is needed. According to RBPF-SLAM framework [20], since the map posterior can be computed analytically given the robot path, it is possible to factorize the joint probability through Rao-Blackwellization [21]:

$$
\operatorname{prob}\left(p_{i, 1: t}^{i 0}, m_{i} \mid z_{i, 1: t}, u_{i, 1: t}^{i 0}\right)=\operatorname{prob}\left(m_{i} \mid p_{i, 1: t}^{i 0}, z_{i, 1: t}\right) \cdot \operatorname{prob}\left(p_{i, 1: t}^{i 0} \mid z_{i, 1: t}, u_{i, 1: t}^{i 0}\right)
$$

In Eq. 4 the state includes the robot trajectory, namely $p_{i, 1: t}^{i 0}=\left\{p_{i, 1}^{i 0}, p_{i, 2}^{i 0}, \ldots, p_{i, t}^{i 0}\right\}$ and the map $m_{i}$, both estimated from the measurements $z_{i, 1: t}$ and the odometric data $u_{i, 1: t}^{i 0}$. The previous equation provides the basis for single robot grid-based RBPFSLAM: the particle filter is applied to the problem of estimating potential trajectories 
and a map hypothesis is associated to each sample. The posterior of robot trajectory is approximated by a set of weighted random samples:

$$
\operatorname{prob}\left(p_{i, 1: t}^{i 0} \mid d_{i, 1: t}^{i 0}\right) \approx \sum_{s=1}^{n} \omega_{t}^{[s]} \delta\left(p_{i, 1: t}^{i 0}-\left\{\bar{p}_{i, 1: t}^{i 0}\right\}^{[s]}\right)
$$

where $n$ is the particle set size, $\left\{\bar{p}_{i, 1: t}^{i 0}\right\}^{[s]}$ is the trajectory of the $s$-th sample at time $t$, $\omega_{t}^{[s]}$ is the corresponding weight $\left(\sum_{s=1}^{n} \omega_{t}^{[s]}=1\right)$, and $\delta(\cdot)$ is the Dirac delta function. Filter prediction is obtained by drawing particles from the proposal distribution $\pi\left(p_{i, t}^{i 0} \mid p_{i, t-1}^{i 0}, d_{i, t}^{i 0}\right)$, which is often derived from a probabilistic description of the motion model of the robot, see [22]. Sample weights are then updated according to [23]:

$$
\omega_{t}^{[s]} \simeq \omega_{t-1}^{[s]} \operatorname{prob}\left(z_{i, t} \mid\left\{\bar{p}_{i, t}^{i 0}\right\}^{[s]}, m_{i}^{[s]}\right), \quad s=1, \ldots, n .
$$

hence using the measurement likelihood to assign importance weight to each particle. Particles degeneracy (i.e., the situation in which most part of the sample set has negligible weight) is then prevented by a resampling phase that randomly chooses the samples which best fit current and past observations, according to particles weights. A common condition for resampling is based on the effective sample size [24], which is an approximated measure of particle diversity:

$$
\tilde{N}_{\text {eff }}=\frac{1}{\sum_{s=1}^{n}\left(\omega_{t}^{[s]}\right)^{2}} .
$$

Particles are re-sampled if the previous quantity drops below a given threshold, usually fixed to $n / 2$, see [25].

Before the first rendezvous, the robot $i$ estimates its belief $\operatorname{prob}\left(p_{i, 1: t}^{i 0}, m_{i} \mid z_{i, 1: t}\right.$, $u_{i, 1: t}^{i 0}$ ) (from the beginning to the current time) given the acquired information $d_{i, 1: t}^{i 0}$. An example of the estimated map and the trajectory hypotheses carried on by the filter is reported in Fig. 2, showing two robots performing single robot RBPF-SLAM. Since the wheel odometry is inaccurate and provides poor motion estimation, several authors proposed to improve the accuracy of relative motion estimation, by using of a scan-matching procedure among laser scans acquired at subsequent poses. This approach is usually referred to as laser-stabilized odometry [17] and it allows to retrieve precise motion estimation while reducing the amount of data to be used for posterior estimation. The scan-matching procedure can be seen as a preprocessing block that discards the redundant data (measures acquired when the robot does not move) and outliers (scans with large matching errors). For further details refer to [17].

\subsection{First Rendezvous and Data Exchange}

Since we made no strict assumption on communication between robots nor on their synchronization, a rendezvous episode, between two generic robots, $i$ and $j$, should be denoted using the time stamp of each robot involved, i.e., $t_{j, k}^{i}$ and $t_{i, k}^{j}$.

At the first rendezvous $(k=1)$ each robot transfers its own piece of information, respectively contained in $d_{i, 1: t_{j, k}^{i}}^{i 0}$ and $d_{j, 1: t_{i, k}^{j}}^{j 0}$, to the teammate, using for example 
Fig. 2 Single robot RBPFSLAM before first rendezvous event. Each robot estimates both trajectory and map hypotheses in its own reference frame
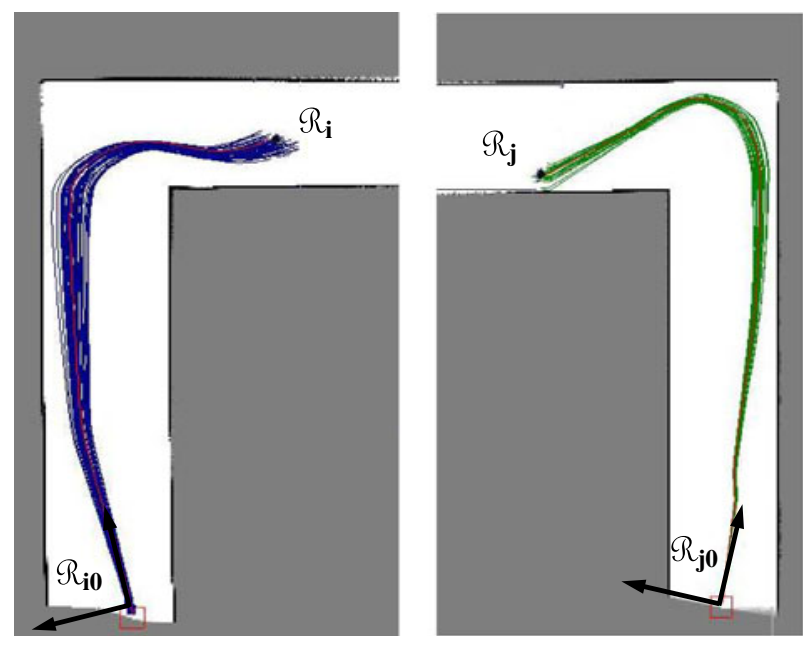

wireless communication or other short range technologies (bluetooth, RFId, etc.). These data contain a list of odometric poses with the corresponding laser scan for each time step, see Eq. 3.

Remark 1 The robots are required to exchange few preprocessed data, and the communication can be limited to rendezvous events. This makes the approach suitable when using an unreliable wireless communication, since, in the short range, the wireless channel is supposed to be more stable, and long distance communications, although useful for enhancing team coordination, are not strictly necessary for our SLAM posterior estimation. The robots can even stop when they meet and wait until a proper connection is established.

For the symmetry of the process and without loss of generality, in the following subsections we will limit our description to robot $i$.

\subsection{Reference Frame Transformation}

When robot $i$ receives $d_{j, 1: t_{i, k}^{j}}^{j 0}$, in order to successfully include this piece of information in its posterior, it is required to transfer the data in its own reference frame, i.e., it has to obtain $d_{j, 1: t_{i, k}^{j}}^{i 0}$. As mentioned in Section 3.2, laser scanner data are expressed in local coordinates, hence only the odometry has to be transformed in $\mathcal{R}_{i 0}$. The coordinate transformation to express $u_{j, 1: t_{i, k}^{j}}^{j 0}$ in $\mathcal{R}_{i 0}$ requires the knowledge of the relative pose between $\mathcal{R}_{i 0}$ and $\mathcal{R}_{j 0}$, i.e., $p_{j 0}^{i 0}$. From Fig. 3 it is possible to observe that $p_{j 0}^{i 0}$ is the composition of the relative poses of four reference frames, i.e., the reference frames of the two robots $\left(\mathcal{R}_{i 0}, \mathcal{R}_{j 0}\right)$ and the reference frames corresponding to the robots poses during rendezvous $\left(\mathcal{R}_{i}, \mathcal{R}_{j}\right)$. Accordingly we can write:

$$
p_{j 0}^{i 0}=p_{i}^{i 0} \oplus p_{j}^{i} \ominus p_{j}^{j 0}
$$


Fig. 3 Reference frames involved in coordinate transformation during a rendezvous event

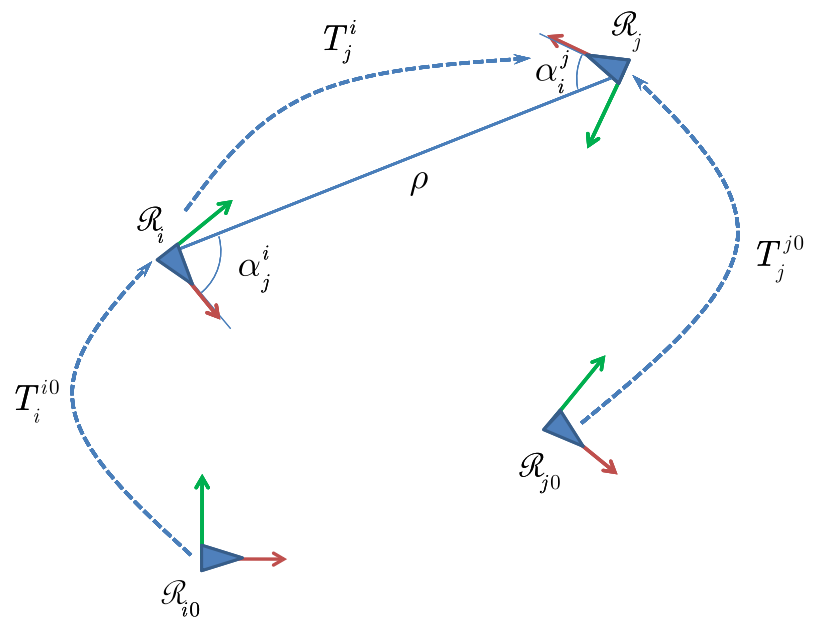

where $p_{i}^{i 0}$ and $p_{j}^{j 0}$ are the poses of the two robot when the meeting occurs, later called rendezvous poses, $p_{j}^{i}$ is the relative pose between the teammates, and $\oplus, \ominus$ are the pose compounding operators [26], see also Remark 3.

In this context we assume that when the robots meet, they are able to measure their relative pose and the corresponding uncertainty using a pan-tilt camera associated to the laser (line of sight between the teammates is required). For each robot, the relative pose of the teammate can be obtained from the relative distance $\rho$ (given by the laser), the angle $\alpha_{j}^{i}$ at which robot $i$ sees the robot $j$, and the angle $\alpha_{i}^{j}$, at which robot $j$ observes robot $i$ (angular measurements can be easily performed through cameras).

Remark 2 The angle $\alpha_{i}^{j}$ and the final odometric pose of robot $j$ in $\mathcal{R}_{j 0}$ should be previously communicated by robot $j$ itself. The distance $\rho$ is measured by the robot $i$, i.e., $\rho=\rho_{j}^{i}$. If $\rho_{i}^{j}$ is also communicated by robot $j$, although not strictly necessary, can be averaged with $\rho_{j}^{i}$ allowing, under the hypothesis of independent Gaussian noise, to reduce the variance of distance measurement to $\sigma_{\rho}^{2}=\sigma_{\rho_{j}^{i}}^{2} \sigma_{\rho_{i}^{j}}^{2} /\left(\sigma_{\rho_{j}^{i}}^{2}+\sigma_{\rho_{i}^{j}}^{2}\right)$.

From the observation of Fig. 4, we notice that the relative angle between the rendezvous poses can be computed using simple geometric considerations:

$$
\theta_{j}^{i}+\alpha_{i}^{j}=\beta=\alpha_{j}^{i} \pm \pi \Longrightarrow \theta_{j}^{i}= \pm \pi+\alpha_{j}^{i}-\alpha_{i}^{j}
$$

Since both signs of $\pi$ represent the relative angle, for sake of simplicity, we consider the plus sign. Hence the relative pose of robot $j$ with respect to robot $i$ becomes:

$$
p_{j}^{i}=\left[\begin{array}{c}
\rho \cos \alpha_{j}^{i} \\
\rho \sin \alpha_{j}^{i} \\
\pi+\alpha_{j}^{i}-\alpha_{i}^{j}
\end{array}\right] .
$$


Fig. 4 When a rendezvous event occurs each robot knows its final pose, respectively expressed in $\mathcal{R}_{i 0}$ and $\mathcal{R}_{j 0}$, and is able to measure the relative pose of the teammate. It is possible to attach a reference frame to the rendezvous pose of each robot in order to understand how the overall transformation is the composition of the rototranslation between the represented reference frames

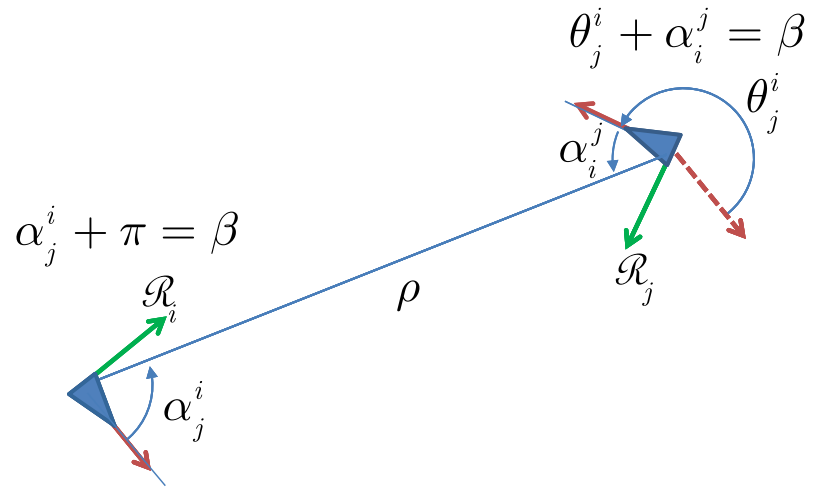

From the previous equation we can further compute the first-order approximation of the relative pose uncertainty, expressed by the covariance matrix $P_{p_{j}^{i}}=\left[P_{m n}\right]$, with $m, n=1,2,3$,

$$
\begin{aligned}
& P_{11}=\sigma_{\rho}^{2} \cos ^{2} \alpha_{j}^{i}+\rho^{2} \sigma_{\alpha_{j}^{i}}^{2} \sin ^{2} \alpha_{j}^{i} \\
& P_{12}=P_{21}=\frac{\sigma_{\rho}^{2}-\rho^{2} \sigma_{\alpha_{j}^{i}}^{2}}{2} \sin \left(2 \alpha_{j}^{i}\right) \\
& P_{13}=P_{31}=-\rho \sigma_{\alpha_{j}^{i}}^{2} \sin \alpha_{j}^{i} \\
& P_{22}=\sigma_{\rho}^{2} \sin ^{2} \alpha_{j}^{i}+\rho^{2} \sigma_{\alpha_{j}^{i}}^{2} \cos ^{2} \alpha_{j}^{i} \\
& P_{23}=P_{32}=\rho \sigma_{\alpha_{j}^{i}}^{2} \cos \alpha_{j}^{i} \\
& P_{33}=\sigma_{\alpha_{j}^{i}}^{2}+\sigma_{\alpha_{i}^{j}}^{2}
\end{aligned}
$$

where $\sigma_{\alpha_{j}^{i}}, \sigma_{\alpha_{i}^{j}}$ and $\sigma_{\rho}$ are the standard deviations of relative angles and distance measurements. From the knowledge of the relative pose and the rendezvous poses, it is possible to compute the vector describing the relative pose between $\mathcal{R}_{i 0}$ and $\mathcal{R}_{j 0}$, according to [8] and [9]. The angle between the two reference frames can be obtained as:

$$
\begin{aligned}
\theta_{j 0}^{i 0} & =\theta_{i, t}^{i 0}+\theta_{j}^{i}-\theta_{j, t}^{j 0}= \\
& =\pi+\alpha_{j}^{i}-\alpha_{i}^{j}+\theta_{i, t}^{i 0}-\theta_{j, t}^{j 0}
\end{aligned}
$$

whereas the Cartesian components of the pose vector can be expressed as:

$$
\begin{aligned}
{\left[\begin{array}{c}
x_{j 0}^{i 0} \\
y_{j 0}^{i 0}
\end{array}\right]=} & {\left[\begin{array}{c}
x_{i}^{i 0} \\
y_{i}^{i 0}
\end{array}\right]+\left[\begin{array}{c}
\rho \cos \left(\theta_{i}^{i 0}+\alpha_{j}^{i}\right) \\
\rho \sin \left(\theta_{i}^{i 0}+\alpha_{j}^{i}\right)
\end{array}\right]+} \\
& +\left[\begin{array}{cc}
\cos \theta_{j 0}^{i 0}-\sin \theta_{j 0}^{i 0} \\
\sin \theta_{j 0}^{i 0} \cos \theta_{j 0}^{i 0}
\end{array}\right]\left[\begin{array}{c}
x_{j}^{j 0} \\
y_{j}^{j 0}
\end{array}\right] .
\end{aligned}
$$

It is worth noticing that each summand in Eqs. 12 and 13 corresponds to a term in Eq. 8, enlightening the role played by each reference frame in the definition of the relative pose between $\mathcal{R}_{i 0}$ and $\mathcal{R}_{j 0}$. The first two components of $p_{j 0}^{i 0}$ correspond to the translation to be applied in order to express the position of robot $j$ in $\mathcal{R}_{i 0}$, 
whereas the last component provides the rotation angle. Hence a generic pose $p_{j, t}^{j 0}=$ $\left[\begin{array}{ll}x_{j, t}^{j 0} & y_{j, t}^{j 0} \\ \theta_{j, t}^{j 0}\end{array}\right]^{\top}$ in $\mathcal{R}_{j 0}$ can be reported in $\mathcal{R}_{i 0}$ through the following transformation:

$$
\left[\begin{array}{c}
x_{j, t}^{i 0} \\
y_{j, t}^{i 0}
\end{array}\right]=\left[\begin{array}{cc}
\cos \theta_{j 0}^{i 0} & -\sin \theta_{j 0}^{i 0} \\
\sin \theta_{j 0}^{i 0} & \cos \theta_{j 0}^{i 0}
\end{array}\right]\left[\begin{array}{c}
x_{j, t}^{j 0} \\
x_{j, t}^{j 0}
\end{array}\right]+\left[\begin{array}{c}
x_{j 0}^{i 0} \\
y_{j 0}^{i 0}
\end{array}\right],
$$

and:

$$
\theta_{j, t}^{i 0}=\theta_{j, t}^{j 0}+\theta_{j 0}^{i 0}
$$

Equations 14 and 15 can be rewritten in compact form using homogeneous coordinates:

$$
\tilde{p}_{j, t}^{i 0}=T_{j 0}^{i 0} \tilde{p}_{j, t}^{j 0}
$$

where:

$$
T_{j 0}^{i 0}=T\left(x_{j 0}^{i 0}, y_{j 0}^{i 0}, \theta_{j 0}^{i 0}\right)=\left[\begin{array}{ccccc}
\cos \theta_{j 0}^{i 0} & -\sin \theta_{j 0}^{i 0} & 0 & x_{j 0}^{i 0} \\
\sin \theta_{j 0}^{i 0} & \cos \theta_{j 0}^{i 0} & 0 & y_{j 0}^{i 0} \\
0 & 0 & 1 & \theta_{j 0}^{i 0} \\
0 & 0 & 0 & 1
\end{array}\right] .
$$

Hence in order to transform $u_{j, 1: t_{i, k}^{j}}^{j 0}$ in $u_{j, 1: t_{i, k}^{j}}^{i 0}$ we apply:

$$
\tilde{u}_{j, 1: t_{i, k}^{j}}^{i 0}=T_{j 0}^{i 0} \tilde{u}_{j, 1: t_{i, k}^{j}}^{j 0} .
$$

Once the stabilized odometry of robot $j$ is roto-translated into the reference frame of robot $i$, the latter has all the necessary information to evaluate SLAM posterior including received data.

Remark 3 The use of homogeneous coordinates is quite unusual in planar transformations. Nevertheless, in the analyzed case, a planar rotation, which represents a mapping $\mathbb{R}^{2} \longrightarrow \mathbb{R}^{2}$, is insufficient to describe the transformation of the three dimensional state space, which includes both position and orientation of the robot. The latter, instead, can be easily treated with homogeneous coordinates, exploiting the analogies between the considered problem and rigid transformations in $3 D$ space. We further observe that composition rules used for $3 D$ roto-translations still apply to our case. Hence we can rewrite Eq. 8 by exploiting the transformation corresponding to each term:

$$
\begin{aligned}
T_{j 0}^{i 0} & =T_{i}^{i 0} T_{j}^{i}\left(T_{j}^{j 0}\right)^{-1}= \\
& =T\left(x_{i}^{i 0}, y_{i}^{i 0}, \theta_{i}^{i 0}\right) T\left(x_{j}^{i}, y_{j}^{i}, \theta_{j}^{i}\right)\left(T\left(x_{j}^{j 0}, y_{j}^{j 0}, \theta_{j}^{j 0}\right)\right)^{-1} .
\end{aligned}
$$

Developing Eq. 19 we obtain analogous results as Eqs. 14 and 15.

Remark 4 The data received were preprocessed by robot $j$, which refined odometry through laser stabilization [17]. Such a preliminary computation reduces the number of recorded poses, since outliers or successive poses in which the robot was stationary are discarded. This fact further shrinks the communication overhead. 
Remark 5 If a wireless communication is active in the considered scenario, the robots can exchange information also in the interval of time between two meetings. In particular the robots can share their current poses, which are fundamental, once the robots know the transformation between their reference frames, in order to plan active rendezvous. Notice that rendezvous remains central, since the odometric information transferred between the robots gradually derives, whereas when meeting occurs pose constraints are added as clarified in the next subsection.

\subsection{SLAM Posterior Estimation on Virtual Data}

In order to obtain grid-based SLAM posterior, for the single robot case, RBPF use the odometric information for the prediction phase, and the measurements from laser scanner for filter update. With respect to Eq. 3 we can state that at each time, as new odometric and laser measures are acquired by robot $i$, one more column is added to the matrix $d_{i, 1: t}^{i 0}$, and this column provides the input for filter estimation one step ahead. In particular, the prediction phase of the filter is based on the change of odometric pose between time $t$ and time $t+1$. Hence the prediction only exploits the odometric constraint given by two subsequent odometric poses, i.e., it only depends on the difference between $u_{i, t+1}^{i 0}$ and $u_{i, t}^{i 0}$, and from its covariance matrix, given by the uncertainty of odometry. Analogously the update phase only depends on laser measurements and the corresponding uncertainty.

We extend the estimation process to the multi robot case by introducing the concept of virtual data. When a rendezvous event occurs, instead of acquiring a single piece of information from odometry and laser, the robot acquires a large amount of information, which includes the past acquisitions of the met teammate. Hence robot $i$ acquires virtual measurements and odometry from robot $j$, gaining knowledge on places that were not physically visited. As a consequence, instead of adding one single column to $d_{i, 1: t_{j, 1}^{i}}^{i 0}$, it concatenates all the data received $d_{j, 1: t_{i, 1}^{j}}^{j 0}$, suitably roto-translated, obtaining:

$$
d_{i, 1: t_{j, 1}^{i}+t_{i, 1}^{j}}^{i 0}=\left[\begin{array}{cccccccc}
u_{i, 1}^{i 0} & u_{i, 2}^{i 0} & \cdots & u_{i, t_{j, 1}^{i}}^{i 0} & u_{j, t_{i, 1}^{i}}^{i 0} & \cdots & u_{j, 2}^{i 0} & u_{j, 1}^{i 0} \\
z_{i, 1} & z_{i, 2} & \cdots & z_{i, t_{j, 1}^{i}} & z_{j, t_{i, 1}^{j}} & \cdots & z_{j, 2} & z_{j, 1}
\end{array}\right] .
$$

It is worth observing that the data are included in reverse order and this is not an arbitrary choice but is due to problem constraints. In a real scenario a rendezvous event imposes a constraint on rendezvous poses, whereas the initial poses of each robot remain unobserved, and can only be inferred from the rendezvous poses and odometric data. In our approach this rendezvous constraint can be exploited applying a suitable prediction model from $u_{i, t_{j, 1}^{i}}^{i 0}$ to $u_{j, t_{i, 1}^{i}}^{i 0}$. When passing from column $t_{j, 1}^{i}$ to $t_{j, 1}^{i}+1$ in Eq. 20, the prediction is not due to a physical movement of the robot but is due to relative pose measurements. As a consequence the odometric constraint is substituted by the rendezvous constraint expressed by the relative pose of the two robots $p_{j}^{i}$ and the corresponding covariance matrix $P_{p_{j}^{i}}$. The estimation over the remaining data is performed according to RBPF-SLAM since between each column an odometric constraint (given by robot $j$ ) holds. When RBPF end the estimation over the odometric path of robot $j$, each particle restarts from its pose at time $t_{j, 1}^{i}$ and 
all particle poses are predicted one step ahead according to the odometry of robot $i$, as in single robot RBPF-SLAM.

We further explain the approach by means of a simple example. In Fig. 5, the initial positions of robot $i$ and $j$ are $S_{1}$ and $S_{2}$, whereas points $F_{1}$ and $F_{2}$ are their rendezvous poses respectively. Therefore, the procedure corresponds to attach the inverted odometric data (from $F_{2}$ to $S_{2}$ ) to the initial odometric data carried on by robot $i$ (from $S_{1}$ to $F_{1}$ ). This piece of information can be used as input to RBPF, that extract the SLAM posterior from the rough data. When applying the prediction step from the last pose of $i\left(F_{1}\right)$ to the final pose of $j\left(F_{2}\right)$, a proper prediction model is considered. The update model remains unaltered since measurements are always given by the laser. After this step the filter continues using the odometry for prediction since the poses from $F_{2}$ back to $S_{2}$ are linked by odometric constraints. When RBPF end the estimation over the path of robot $j$, each particle restarts from its rendezvous pose and particles are predicted one step later to $S_{3}$. Hence the robot continues the estimation through RBPF-SLAM applied to its own measurements until the exploration process ends at a generic point $F_{3}$.

During the estimation over the external data, the robot $i$ processes the information of the other robot as it was traveling backward following the trajectory of the robot $j$. The surplus of information $d_{j, 1: t_{i, 1}^{j}}^{i 0}$ represents a kind of virtual movement, since the robot $i$ acquires measurements on the environment and on the odometric poses of robot $j$ that were not obtained physically from its own sensors but were observed and communicated by another robot. After the rendezvous, robot $i$ posterior $\operatorname{prob}\left(p_{i, 1: t_{j, 1}^{i}+t_{i, 1}^{j}}^{i 0}, m_{i} \mid d_{i, 1: t_{j, 1}^{i}}^{i 0}, d_{j, 1: t_{i, 1}^{i}}^{i 0}\right)$ includes the data of robot $j$ and both the map and the trajectories are updated accordingly.

Finally we must observe that the approach is effective since the estimation process is remarkably faster than the acquisition of new measurements. As a consequence the information carried on by the other robot are quickly included in the posterior,

Fig. 5 Multi robot RBPF-SLAM. After rendezvous the overall map and trajectory hypotheses include the information acquired by both robots involved in the meeting

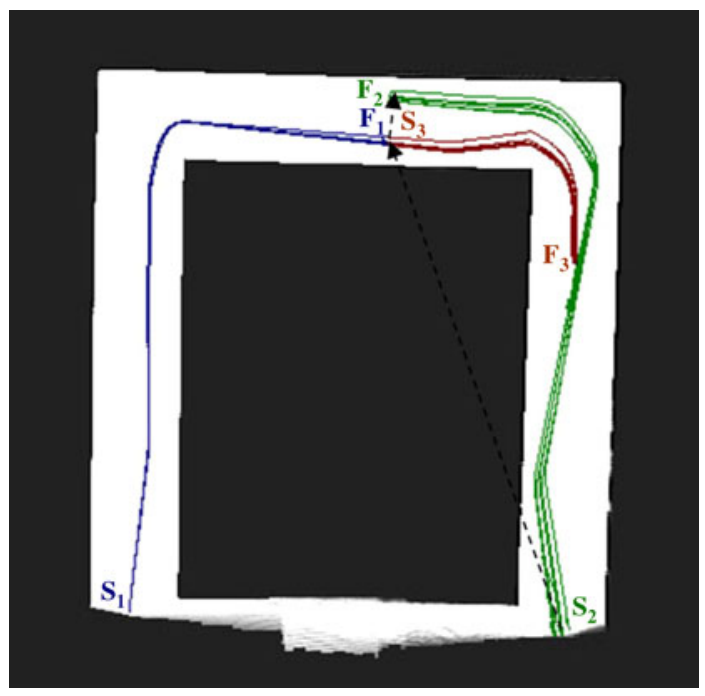


preserving the on-line nature of the estimation process. Details on latencies are reported in Section 4.

\subsection{Following Rendezvous Events}

The procedure described in the previous subsection can be easily generalized to an arbitrary number of meetings. After the first rendezvous each new meeting with a previously met robot corresponds to a loop closing event, adding constraints that are introduced in the filter through a resampling phase which selects the trajectories that best describe all the information acquired. Moreover, including virtual data from other robots, loop closure can occur also if the robot revisits places traveled by the met teammates. At the $k$-th rendezvous, robot $j$ does not transfer the entire data $d_{j, 1: t_{i, k}^{j}}^{j 0}$ but only the piece of information from the last meeting to the current time stamp, i.e., $d_{j, t_{i, k-1}^{j}: t_{i, k}^{j}}^{j 0}$ This is not only a necessity dictated by the limited bandwidth, but derives from structural properties of the filtering process. If the same data are included twice in the RBPF, the filter interprets this information as the robot traveled twice in a place that was actually visited only once. As a consequence resampling phases occur although no useful information for resampling is added. Based on this consideration our method allows to preserve filter consistency and at the same time it takes advantage of the small amount of data exchanged during rendezvous.

Remark 6 (Application to heterogeneous teams) We focused on the very specific case of robots equipped with a laser scanner since this setup is widespread for indoor mobile robotics applications, for laser capability of acquiring accurate range information over long distances. In smaller scenarios, however, one can envision to apply the same approach with other distance sensors, as ultrasound sensors [27] or cameras [28], by simply choosing a suitable sensor model. Moreover the team can be heterogeneous: in such a case the communication between agents should include further information on robot sensor models, which have to be applied when performing estimation over virtual data (see Section 3.7). Also the choice of cameras (and distance measurements from laser scanner) for relative pose estimation can be easily substituted by other technological solutions, thus reducing to a similar probabilistic treatment, under a suitable uncertainty propagation (see Section 3.6). For instance the literature on relative pose estimation includes the use of vision based techniques [29] or the inference of relative pose from multiple distance measurements [30].

We conclude this section observing that when more than two robots intervene in the estimation process the procedure described above remains unchanged. The only aspect to be carefully considered is the imposition of the constraints given by the odometry, measurements and rendezvous events. In our implementation we preferred each robot to provide only the information acquired through its own sensors, regardless past meetings with other teammates. Roughly speaking the robot does not transfer virtual data received in previous meetings, since in such a case, the constraints would become difficult to manage. In this fashion we preserve the simplicity of implementation making the proposed technique an effective extension of grid-based RBPF-SLAM. 
The proposed approach is validated and discussed in the following section in which both simulations and experimental results are presented.

\section{Test and Discussion}

In this section we report the results of the implementation of our approach in simulations and real scenarios. Both virtual and real tests were carried out using the adaptive resampling technique, proposed by Stachniss et al. [25], and stabilized laser odometry, further detailed in [17]. The mobile robots used are ActivMedia Pioneer P3-DX equipped with laser range sensor SICK LMS200, a LOGITECH pantilt camera and odometry pose estimation. The scenario is an indoor environment, in which the robots travel for the purpose of building a consistent and shared map.

\subsection{Multi Robot Simulations}

Simulations were performed in MobileSim, a real time simulator, used to test the approach in realistic environments, with an arbitrary number of robots. Before introducing some results from the simulated multi robot system we underline the importance of considering the uncertainty in the relative pose measurement, when dealing with multi robot SLAM. In Fig. 6 a simple example of RBPF multi robot SLAM is reported. Robot 1 starts from point $S_{1}$ and meets robot 2 in $I_{1}$, receiving data from the latter. Then robot 1 continues its estimation until it arrives in a generic position $F_{1}$. We limit this simple example to robot 1 perspective. In Fig. 6a we consider the error on relative measurement and the resulting map of the corridor, estimated from data of both robots, is achieved in a correct manner. In Fig. $6 b$ we

Fig. 6 Toy example of multi robot RBPF-SLAM. In (a) the uncertainty in relative measurement is taken into account, whereas it is neglected in (b)

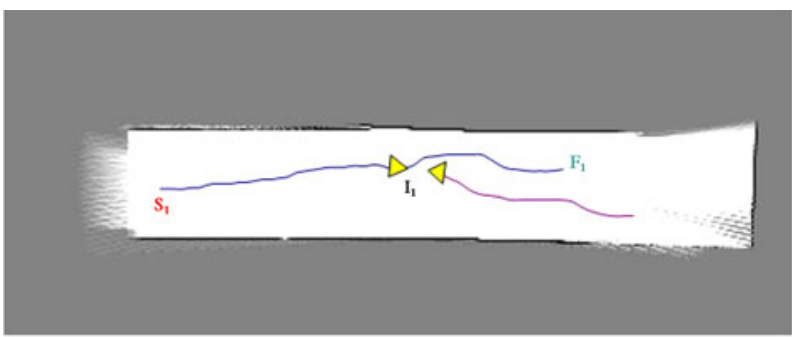

(a)

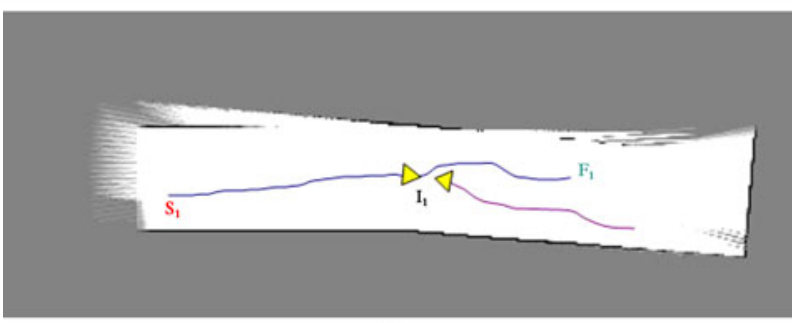

(b) 
neglect the uncertainty in the relative measurement and, as expected, the underestimation of the uncertainty leads to an inconsistent map. This phenomenon worsens in real tests in which the error affecting measurements is even bigger due to sensor calibration errors and measurements noise.

After these preliminaries we present the results of simulations in a logistic space. Three robots travel in the environment, and meetings occur randomly when they are in reciprocal field of view and their distance is less than 3 meters (for simplicity teleoperation is used for moving the agents since in this context we are not interested in the particular exploration policy). During each rendezvous event, robots exchange data and they are able to estimate the map from received information. At the end of the process each robot has the overall map, built in a cooperative fashion. In Fig. 7 the estimated maps are shown. Figure 7 a reports the trajectory of robot 1 and the map built by the robot neglecting the rendezvous events: robot 1 starts in the position denoted with the red circle, ending its exploration in the place labeled with the green square. In such a case the agent performs single robot SLAM and it is able to recover only a partial representation of the environment. Figure $7 \mathrm{~b}$ shows the case in which the robot takes advantage of the rendezvous episodes (according to our approach) and is able to include the information acquired by the teammates in its SLAM posterior. The corresponding information for robot 2 and 3 are shown in Fig. $7 \mathrm{c}, \mathrm{d}$ and e, f, respectively. Figure 7c and e report the single robot SLAM outcome and the trajectories followed by the agents, whereas Fig. $7 \mathrm{~d}$ and f show the estimated map with the multi robot approach. We remark that the trajectories followed by the agents, in the multi robot SLAM case, are identical to the corresponding trajectories shown in Fig. 7a-e.

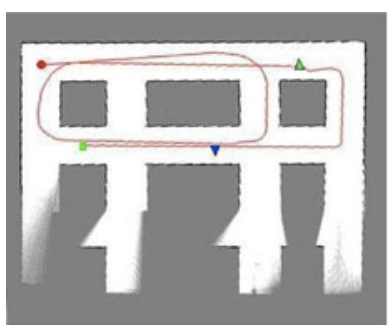

(a)

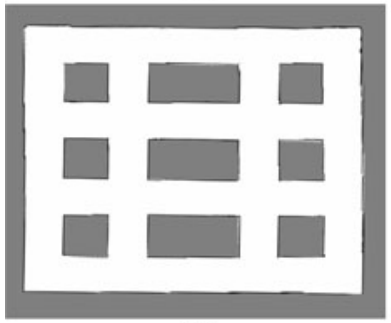

(b)

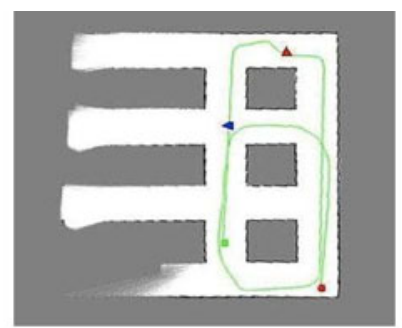

(c)

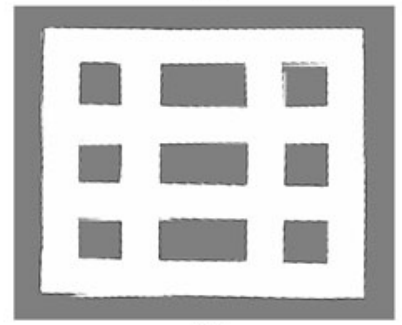

(d)

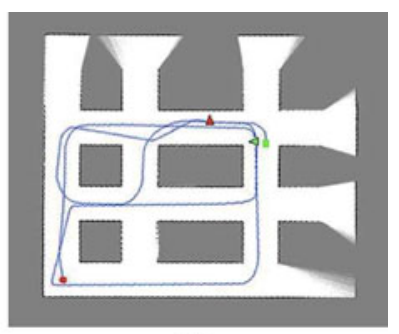

(e)

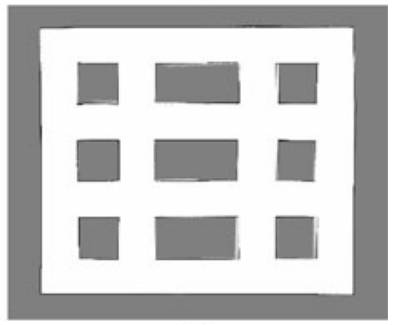

(f)

Fig. 7 Comparison of the maps of the simulated logistic space, obtained by each agent in the single robot case (a), (c), (e), and with the proposed multi robot approach (b), (d), (f). The trajectories of the robots are shown in the upper sub-figures, whereas the red circles, the green squares and the triangles denote the starting points, the final positions and the rendezvous positions for each agent 
Remark 7 The approach is distributed and for the nature of the RBPF-SLAM, it leads to non overlapping representations of the environment among the robots. Although the maps built by each teammate are not exactly equal, due to the random nature of the particle filter, they only differ by few cells, preserving the structure of the scenario. As a consequence our approach allows to build a shared map which can be used for team coordination.

In order to give quantitative evidence of the similarity between the maps of the robots after the end of the SLAM process, we now compare these grid maps with a ground truth map, estimated by a single robot that visits all the scenario. The test scenario is challenging, since the robot is requested to cover an area of $1,100 \mathrm{~m}^{2}$, producing an "ideal" map to be used as ground truth. The single robot map and the corresponding trajectory followed by the agent are shown in Fig. 8.

Overcoming a simple visual inspection, we use the metric proposed by Carpin in [14]. We report the following definition from the cited paper.

Definition 1 Let $M_{1}$ and $M_{2}$ be two grid maps. The agreement between $M_{1}$ and $M_{2}$ (indicated as $\operatorname{agr}\left(M_{1}, M_{2}\right)$ ) is the number of cells in $M_{1}$ and $M_{2}$ that are both free or both occupied. The disagreement between $M_{1}$ and $M_{2}$ (indicated as $\operatorname{dis}\left(M_{1}, M_{2}\right)$ ) is the number of cells such that $M_{1}$ is free and $M_{2}$ is occupied or vice-versa. The acceptance index between them is defined as:

$$
\omega\left(M_{1}, M 2\right)=\left\{\begin{array}{cl}
0 & \text { if } \operatorname{agr}\left(M_{1}, M_{2}\right)=0 \\
\frac{\operatorname{agr}\left(M_{1}, M_{2}\right)}{\operatorname{agr}\left(M_{1}, M_{2}\right)+\operatorname{dis}\left(M_{1}, M_{2}\right)} & \text { if } \operatorname{agr}\left(M_{1}, M_{2}\right) \neq 0
\end{array}\right.
$$

The acceptance index gives information on map similarity, once a suitable roto-translation is applied (in our approach each robot preserves its initial reference frame). Rototranslation is computed according to [14]. Table 1 reports the

Fig. 8 Map of the logistic space estimated with RBPF-SLAM (single robot). This grid map is used as ground truth and compared with the maps estimated with the proposed multi robot SLAM approach

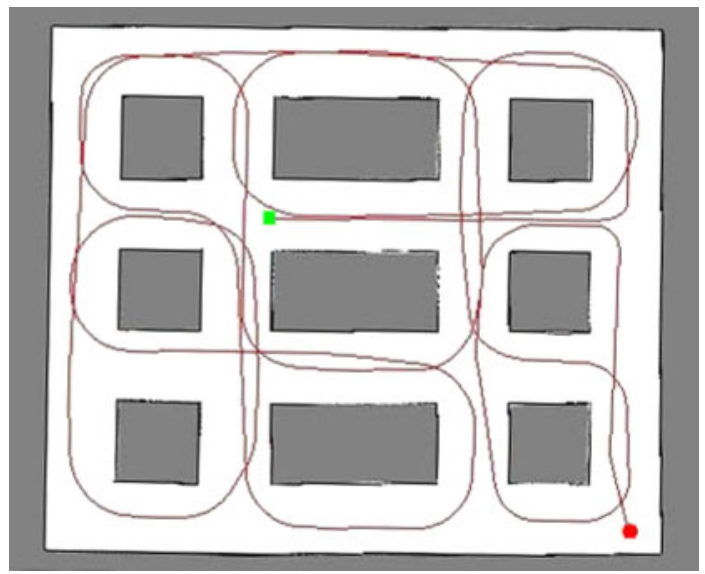


Table 1 Acceptance index of the three maps cooperatively built by the robots with respect to the map estimated through single robot RBPF-SLAM

\begin{tabular}{llll}
\hline & Robot 1 & Robot 2 & Robot 3 \\
\hline Acceptance index & 0.96 & 0.95 & 0.97 \\
\hline
\end{tabular}

acceptance index values of the maps estimated cooperatively by the three robots (see Fig. 7b-f), with respect to map estimated by the single robot (see Fig. 8). The high values of the acceptance index indicates the consistency of the maps produced by the multi robot system and their similarity.

Figure 9 shows the length of the sensor data queue that should be processed at each time step by one of the three robots. This length coincides with the number of columns of Eq. 20 waiting for being included in the posterior. The $x$-axis of the figure corresponds to physical time. Notice that the two peaks, that coincide with the instant in which external data are received from the other teammates, are quickly shortened by the RBPF. This observation allows us to conclude that, after a latency, the estimation process comes back to its on-line nature. The maximum delay observed, using common laptop, was $190 \mathrm{~s}$. The delay is proportional to the peak height (approximately given by the amount of data received), hence, frequent meetings lead to lower peaks and shorter latencies, because agents are required to exchange only the data acquired after the last meeting. We notice that the experiment reported here is a worse case example, since the robots travel independently for a long time and meet after exploring large areas, hence making the communication more demanding. A more common situation is the one reported in Section 4.2 (see Fig. 12 for comparison), which further highlights that the approach is sustainable and suitable for long operation.

We conclude this section with some results on the practical advantages of our technique with respect to single robot SLAM approach. In Table 2 we report the distance traveled by each robots in the multi robot SLAM case (i.e., the length of the trajectories shown in Fig. 7a-e) and the length of the path followed by the agent

Fig. 9 Length of the sensor data queue that should be processed at each time step of simulation. The peaks correspond to rendezvous events in which external data are added to the queue

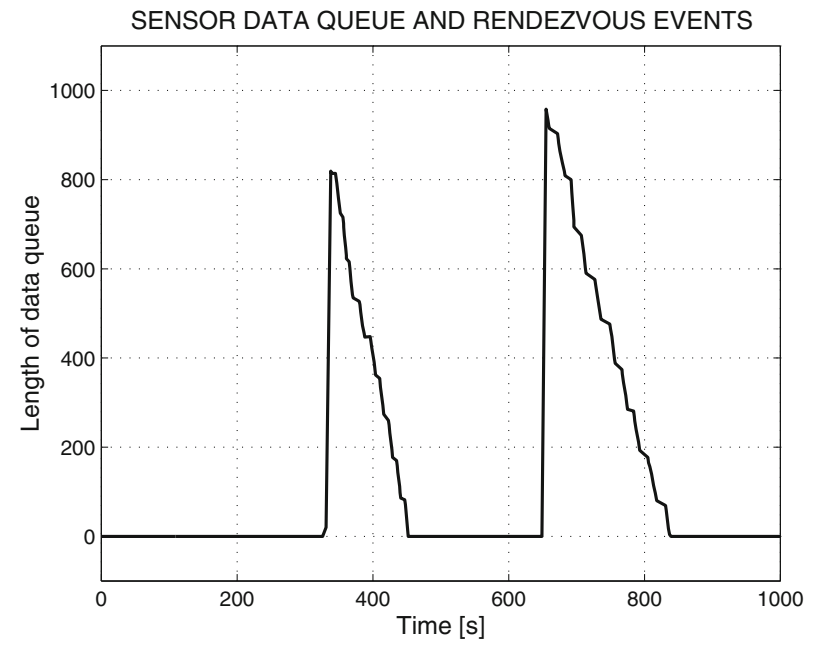


Table 2 Distance traveled by the robots and time required for building the overall map, in the multi robot case and in the single robot scenario

\begin{tabular}{llllr}
\hline & Multi robot & & Single robot \\
\cline { 2 - 4 } & Robot 1 & Robot 2 & Robot 3 & \\
\hline Time (s) & 805 & 795 & 992 & 1,425 \\
Distance traveled (m) & 128 & 113 & 183 & 402 \\
\hline
\end{tabular}

in the single robot SLAM case, see Fig. 8. It is possible to verify that the overall distance traveled by the team (i.e., the sum of the distances traveled by each robot in the multi robot system) is similar to the one traveled by the single robot, but since such distances are traveled in parallel, the overall estimation process is speeded up. Evidence of such consideration is reported in the same table, in which we also show the time required to build the complete map in the multi robot case (for each agent) and in the single robot case. It is quite intuitive that the advantage of the information exchange relies on the capability of the robots of estimating the whole map, without spending time in acquiring sensor measurements. Hence, in our experiments, after less than $1,000 \mathrm{~s}$ all the robots of the team have a complete map representation, whereas a robot alone, would require about 1,500 s.

\subsection{Real Tests}

In this section we report the results of the implementation of our approach in two real scenarios. The first experiment is useful to further explain the approach and describe its real implementation. The second, instead, is an application of SLAM in an artificially created environment, that reproduces the structure of a logistic space.

We firstly considered the case in which two robots travel inside an office-like environment cooperatively building a map. This experiment was performed in the corridors and labs of Politecnico di Torino. The test scenario is challenging since it was performed in an environment with many non reflective surfaces in presence of people traversing corridors. The mobile robots are shown in Fig. 10. Each teammate

Fig. 10 Robots P3-DX used for real tests. A bar code marker is used to distinguish the robots

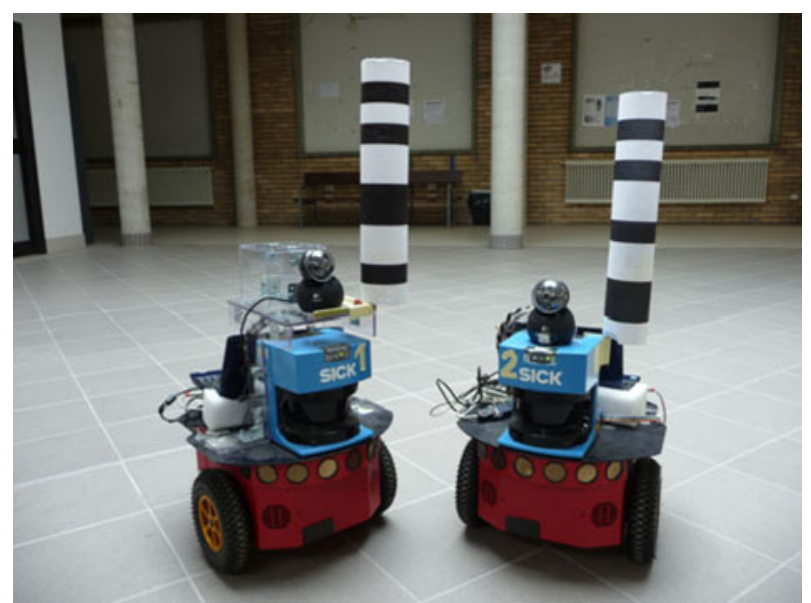


is equipped with a visual marker, and this marker is endowed with a bar code useful to distinguish the robots. The map estimated by robot 1 and the corresponding estimated trajectory (including the pieces of data received from robot 2) are shown in Fig. 11. The reader is referred to the same figure for the following description.

The two teammates are initially deployed in separated locations, respectively labeled with $S_{1}$ and $S_{2}$. The robots cover the first piece of trajectory until they arrive in positions $I_{1}$ and $I_{2}$, where the first rendezvous occurs. Once the robots meet, they measure the relative poses and exchange data using a wireless communication (based on a client/server architecture). Robot 1 includes in its posterior the external information related to the path $S_{2}$ to $I_{2}$ of robot 2 and then continues its route, traveling in loop (A) and applying RBPF-SLAM. In the meanwhile robot 2 explores the lab (B) and arrives in position $F_{2}$. Robot 1 visits room (C) and, once arrived in $F_{1}$, it finally meets robot 2 for the second time. The data received from robot 2 allow robot 1 to complete its map, reducing the time required for exploration and enhancing loop closing. The dual procedure is applied by robot 2 , producing a similar map.

In Fig. 12 we report the length of data queue of an agent: in this case the first rendezvous occurred after the robots have explored a small area, hence the peak is low and the virtual data is processed by the agent in few seconds. During the second meeting, instead, the maximum observed latency was less than $40 \mathrm{~s}$.

The second experiment was lead in an environment of approx. $300 \mathrm{~m}^{2}$ reproducing the structure of a logistic space. In Fig. 13a the map estimated by robot 1 is shown. The figure also shows robot trajectory, in which the red circle denotes robot starting point, yellow triangles correspond to places in which a rendezvous occurred and

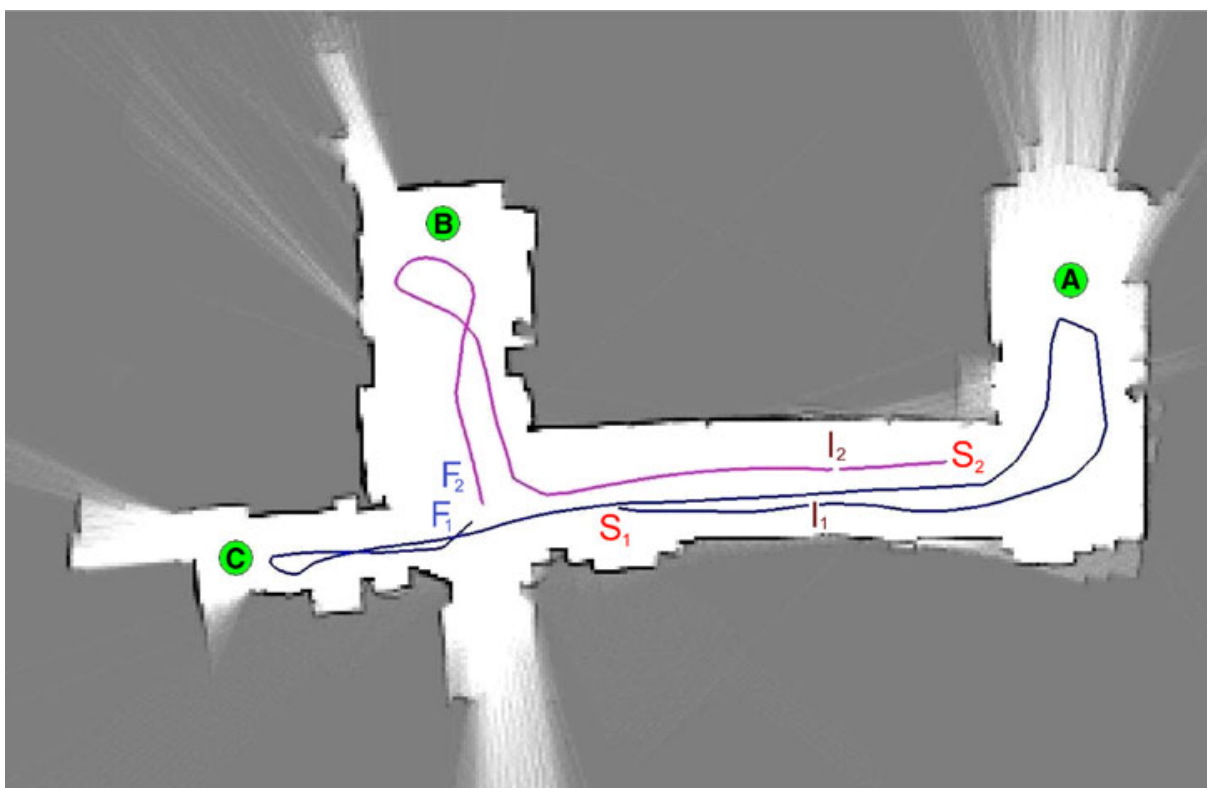

Fig. 11 Map estimated through RBPF multi robot SLAM during experimental test at Politecnico di Torino. The corridor length is about $20 \mathrm{~m}$ whereas the particle set size was 100 
Fig. 12 Length of the sensor data queue that should be processed at each time step of the real test. The peaks correspond to rendezvous events in which external data is added to the queue

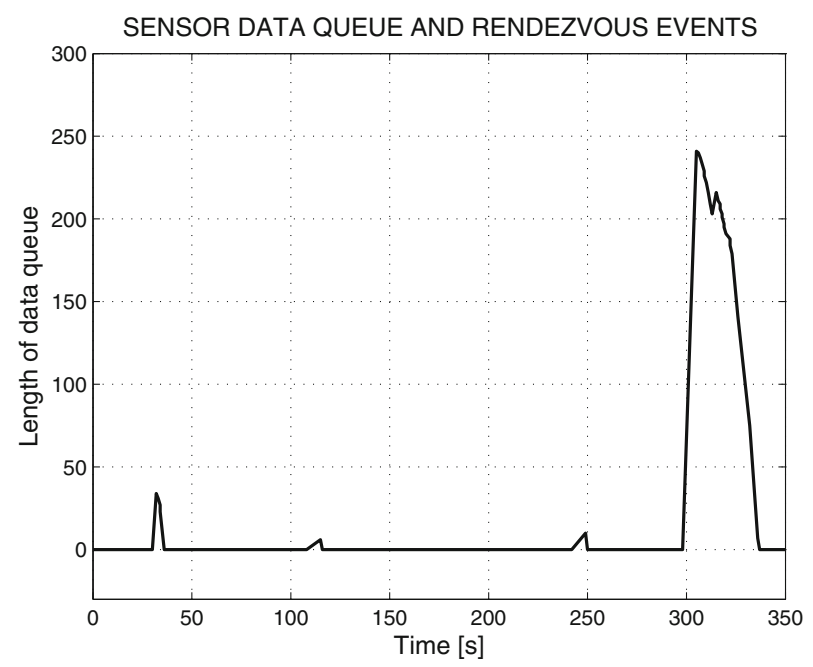

green square corresponds to the end of robot 1 trajectory. Figure $13 \mathrm{~b}$ reports the same information with respect to robot 2. According to our approach the robots can build a similar representation of the environment in a distributed fashion without

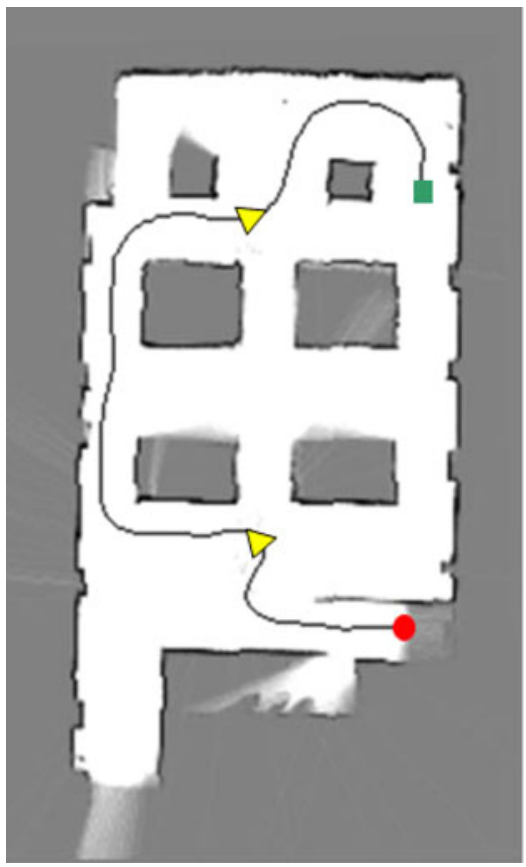

(a) Robot 1

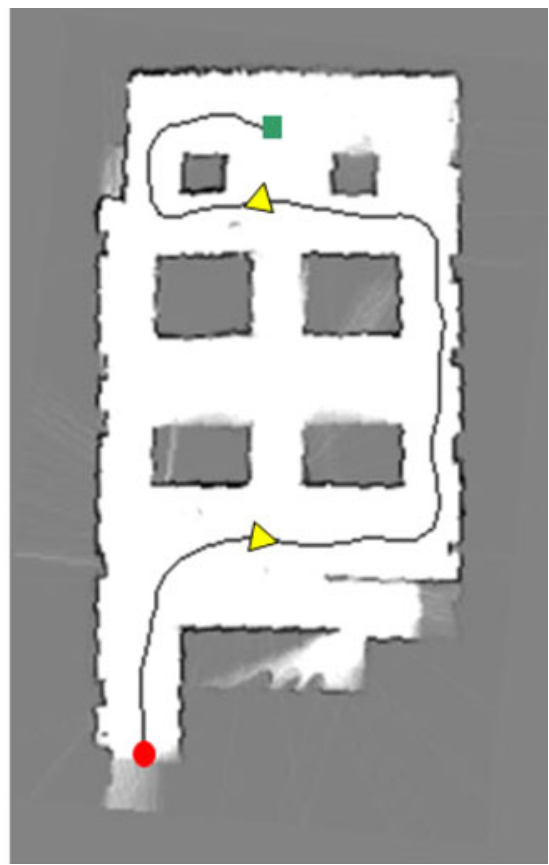

(b) Robot 2

Fig. 13 Maps estimated by the two Pioneer P3-DX during the real test in logistic space-like environment. Figures show robot trajectory (red circle is the starting point, green square is the end of the path) and the rendezvous points (yellow triangles) 
physically traveling in the whole scenario. The acceptance index between the two maps is equal to 0.92 .

We further stress that no synchronization is needed and the approach requires no central computation, constituting a fully distributed multi robot SLAM solution. The formation of robots can be seen as a mobile sensor network, in which a communication link appears between two nodes, only during rendezvous events. In such a way fault tolerance problems in communication, as investigated in [27], are relaxed and also in case the robots fail to detect a rendezvous case, this does not influence the estimation process. These considerations remark the advantages over the state of the art approaches. The robots are no longer constrained by the communication ranges of the teammates as in [17], but can proceed in their exploration independently. Moreover the approach takes into consideration the uncertainty on relative measurements, whereas neglecting these errors can lead to an underestimation of filter uncertainty, thus to inconsistencies in the occupancy grid map. Finally each robot treats the virtual data as its own measurements, and the filter is no longer required to perform estimation over an augmented state (see [17]), hence avoiding the use of large sample size (the number of required samples increases exponentially in the number of state variables [31]).

\section{Conclusion}

As multi robot systems are envisioned to play an important role in many robotic applications, distributed techniques for solving Simultaneous Localization and Mapping are required, in order to enhance autonomous exploration and large scale SLAM, increasing both efficiency and robustness of operation. Although RaoBlackwellized Particle Filters (RBPF) have been demonstrated to be an effective solution to the problem of single robot SLAM, few extensions to teams of robots exist, and these approaches are characterized by strict assumptions on both communication bandwidth and prior knowledge on relative poses of the teammates. The present paper proposes an efficient extension of RBPF-SLAM to the multi robot scenario. We relaxed the assumptions of related works, addressing the problem of multi robot SLAM in the case of limited communication and unknown relative initial poses. Our approach allows to jointly estimate SLAM posterior of the robots by fusing the prioceptive and the eteroceptive information exchanged among teammates. RBPF multi robot SLAM involves the communication of a small amount of data, while taking into account the uncertainty in relative pose measurements. Moreover it can be naturally extended to different communication technologies (bluetooth, RFId, Wifi, etc.) regardless their sensing range. Before and after each rendezvous the robots of the team perform their estimation using RBPF-SLAM. When a rendezvous occurs, a simple procedure allows to enhance information fusion in an effective and distributed fashion. This procedure can be summarized in three phases, respectively called data exchange, reference frame transformation and estimation on virtual data. When the filtering of received data is complete, the particles restart from their poses before the meeting, and continue the estimation process, according to gridbased RBPF-SLAM. At the end of the mapping process the robots share similar (i.e., not exactly equal) representations of the map, up to a known roto-translation. Overcoming the visual inspection that is often applied for result evaluation we use 
the acceptance index proposed by Carpin in [14] to provide an objective evaluation of the estimation process. After the first meeting the robots know the relative transformation between the reference frames of the met teammates, enhancing the possibility of team coordination and active rendezvous. The technique is shown to be an effective solution to multi robot SLAM and it is further validated through simulations and real tests. Real experiments were performed in office-like environment and in an artificially built scenario which reproduces the structure of a logistic space.

Acknowledgements This work was supported by the research grant from REGIONE PIEMONTE, BANDO 2006, RICERCA INDUSTRIALE E SVILUPPO PRECOMPETITIVO, MACP4LOG "Mobile, autonomous and cooperating robotic platforms for supervision and monitoring of large logistic surfaces" (RU/02/26).

Open Access This article is distributed under the terms of the Creative Commons Attribution Noncommercial License which permits any noncommercial use, distribution, and reproduction in any medium, provided the original author(s) and source are credited.

\section{References}

1. Siciliano, B., Khatib, O.: Springer Handbook of Robotics. Springer (2008)

2. Fox, D.: Distributed multirobot exploration and mapping. In: Proc. of the 2nd Canadian Conference on Computer and Robot Vision (2006)

3. Durrant-Whyte, H., Bailey, T.: Simultaneous localisation and mapping (SLAM): part I. The essential algorithms. Robot. Autom. Mag. 13, 99-110 (2006)

4. Durrant-Whyte, H., Bailey, T.: Simultaneous localisation and mapping (SLAM): part II. State of the art. Robot. Autom. Mag. 13, 108-117 (2006)

5. Thrun, S.: A probabilistic online mapping algorithm for teams of mobile robots. Int. J. Rob. Res. 20(5), 335-363 (2001)

6. Williams, S., Durrant-Whyte, H.: Towards multi-vehicle simultaneous localisation and mapping. In: Proc. of the IEEE International Conference on Robotics and Automation (2002)

7. Stachniss, C.: Robotic mapping and exploration. In: Springer Tracts in Advanced Robotics, vol. 55. Springer (2009)

8. Zhou, X.S., Roumeliotis, S.I.: Multi robot SLAM map alignment with rendezvous. Technical Report, 2005-001 (2005)

9. Zhou, X.S., Roumeliotis, S.I.: Multi-robot SLAM with unknown initial correspondence: the robot rendezvous case. In: Proc. of IEEE/RSJ International Conference on Intelligent Robots and Systems, pp. 1785-1792 (2006)

10. Spanos, D.P., Olfati-Saber, R., Murray, R.: Approximate distributed Kalman filtering in sensor networks with quantifiable performance. In: Proc. of the 4th International Symposium on Information Processing in Sensor Networks, vol. 760, pp. 133-139 (2005)

11. Spanos, D.P., Olfati-Saber, R., Murray, R.: Distributed sensor fusion using dynamic Consensus. In: Proc. of the 16th IFAC World Congress (2005)

12. Thrun, S., Liu, Y.: Multi-robot SLAM with sparse extended information filters. In: Springer Tracts in Advanced Robotics, vol. 15, pp. 254-266 (2005)

13. Chang, H., Lee, C., Hu, Y., Lu, Y.: Multi-robot SLAM with topological/metric maps. In: Proc. of the IEEE/RSJ International Conference on Intelligent Robots and Systems (2007)

14. Carpin, S.: Fast and accurate map merging for multi-robot systems. Auton. Robots 25, 305-316 (2008)

15. Howard, A., Sukhatme, G.S., Mataric, M.J.: Multirobot simultaneous localization and mapping using manifold representations. In: Proc. of the IEEE, vol. 94, pp. 1360-1369 (2006)

16. Gutmann, J., Konolige, K.: Incremental mapping of large cyclic environments. In: Proc. of the 6th IEEE International Symposium on Computational Intelligence in Robotics and Automation, pp. 318-325 (2000)

17. Howard, A.: Multi-robot simultaneous localization and mapping using particle filters. In: Robotics: Science and Systems, pp. 201-208 (2006) 
18. Carlone, L., Kaouk Ng, M., Du, J., Bona, B., Indri, M.: Reverse KLD-sampling for measuring uncertainty in Rao-Blackwellized particle filters SLAM. In: Workshop on Performance Evaluation and Benchmarking for Next Intelligent Robots and Systems. IEEE/RSJ International Conference on Intelligent Robots and Systems (2009)

19. Stachniss, C., Grisetti, G., Burgard, W., Roy, N.: Analyzing Gaussian proposal distributions for mapping with Rao-Blackwellized particle filters. In: Proc. of the International Conference on Intelligent Robots and Systems, pp. 3485-3490 (2007)

20. Grisetti, G., Tipaldi, G.D., Stachniss, C., Burgard, W., Nardi, D.: Fast and accurate SLAM with Rao-Blackwellized particle filters. J. Robot. Auton. Syst. 55, 30-38 (2007)

21. Doucet, A., de Freitas, J., Murphy, K., Russel, S.: Rao-Blackwellized particle filtering for dynamic bayesian networks. In. Proc. of the Conference on Uncertainty in Artificial Intelligence, pp. 176-183 (2000)

22. Thrun, S., Burgard, W., Fox, D.: Probabilistic Robotics. MIT Press (2005)

23. Stachniss, C., Grisetti, G., Burgard, W.: Analyzing Gaussian proposal distributions for mapping with Rao-Blackwellized particle filters. In: Proc. of International Conference on Intelligent Robots and Systems (2007)

24. Arulampalam, S., Maskell, S., Gordon, N., Clapp, T.: A tutorial on particle filter for on-line nonlinear/non-Gaussian bayesian tracking. IEEE Trans. Signal Process. 2(50), 174-188 (2002)

25. Stachniss, C., Grisetti, G., Hahnel, G., Burgard, W.: Improved Rao-Blackwellized mapping by adaptive sampling and active loop-closure. In: Proc. of the Workshop on Self-Organization of Adaptive behavior, pp. 1-15 (2004)

26. Lu, F., Milios, E.: Globally consistent range scan alignment for environment mapping. J. Auton. Robots 4, 333-349 (1997)

27. Mendez Polanco, J.A., Muñoz-Meléndez, A.: Collaborative robots for indoor environment exploration. In: 10th International Conference on Control, Automation, Robotics and Vision (2008)

28. Macchia, V., Rosa, S., Carlone, L., Bona, B.: An application of omnidirectional vision to gridbased SLAM in indoor environments. In: Proc. of the 2010 International Conference on Robotics and Automation, Workshop on Omnidirectional Robot Vision (2010)

29. Sagues, C., Murillo, A.C., Guerrero, J.J., Goedemé, T., Tuytelaars, T., Gool, L.V.: Localization with omnidirectional images using the $1 \mathrm{~d}$ radial trifocal tensor. In: Proc. of the IEEE International Conference on Robotics and Automation, pp. 551-556 (2006)

30. Zhou, X., Roumeliotis, S.: Robot-to-robot relative pose estimation from range measurements. IEEE Trans. Robot. 24(6), 1379-1393 (2008)

31. Khan, Z., Balch, T., Dellaert, F.: A Rao-Blackwellized particle filter for eigentracking. In: Proc. of IEEE Computer Society Conference on Computer Vision and Pattern Recognition (2004) 